\title{
Variability of laser-evoked potentials: attention, arousal and lateralized differences
}

\author{
Ahmad Beydoun, Thomas J. Morrow, Joanne F. Shen and Kenneth L. Casey \\ Department of Neurology, University of Michigan Medical School, Ann Arbor, MI (USA), and Neurology Research Laboratories, \\ Veterans Administration Medical Center, Ann Arbor, MI (USA)
}

(Accepted for publication: 21 December 1992)

\begin{abstract}
Summary We recorded laser-evoked potentials (LEPs) from 20 normal subjects by stimulating the skin with pulses from an infrared $\mathrm{CO}_{2}$ laser. The conduction velocity of the peripheral afferent fibers mediating the LEPs averaged $14.9 \mathrm{~m} / \mathrm{sec}$. The amplitude of the LEP components correlated significantly with perceived stimulus intensity. During repetitive constant intensity stimulation, the peak-to-peak LEP amplitude decreased 38\% during a distraction task and $42 \%$ during drowsiness and was absent during stage 2 sleep, indicating a modulation of responsiveness to laser stimulation during distraction and decreased states of arousal. Normative data revealed considerable intersubject variability in LEP latencies and amplitudes. Analysis of intrasubject lateralized (side-to-side) differences revealed that the relative peak-to-peak amplitude was less variable than that of the $\mathrm{N}$ or $\mathrm{P}$ components. For clinical applications using 3 S.D.s to define the normal range, a lateral interpeak amplitude difference greater than $28 \%$ would suggest focal or lateralized sensory abnormality in an individual patient. Vigilance and attentiveness to the stimuli should be monitored during the acquisition of LEPs.
\end{abstract}

Key words: Laser; Event-related potentials; Pain; Variability; Drowsiness; Sleep; Attention

A neurophysiological correlate of pain in humans would be valuable for basic and clinical research on pain mechanisms and the pathophysiology of abnormal pain. The development of microneuronography, for example, has enabled stimulation and recording from single small-diameter afferent fibers of human subjects while simultaneously obtaining reports of their sensory experiences (Gybels et al. 1979; Vallbo et al. 1979; Adriaensen et al. 1983). Correlations between afferent activity in putative nociceptors and intensity of pain experience have been obtained in some, but not all, experiments (Gybels et al. 1979). Although useful in the analysis of the physiology and pathology of nociceptive afferents, microneuronography can only indirectly assess central nervous system (CNS) nociceptive function; moreover, the technique is invasive, sometimes painful, and demanding of professional time and skill.

In 1976, Carmon et al. first demonstrated that cutaneous stimulation of human subjects with brief pulses from an infrared $\mathrm{CO}_{2}$ laser could evoke a near-field cerebral vertex potential that correlated in amplitude

Correspondence to: Dr. Ahmad Beydoun, University of Michigan Medical Center, University Hospital 1B300/0036, 1500 E. Medical Center Drive, Ann Arbor, MI 48109-0036 (USA).

Tel.: (313) 936-9030. with the intensity of pain sensation. Although similar pain-related cerebral potentials had been reported by others using electrical tooth pulp stimulation (Chatrian et al. 1975; Chapman et al. 1979), cutaneous infrared laser stimulation offered the advantages of stimulus specificity and of allowing the stimulation of different skin sites. Heat pulses generated by a laser induce a rapid rise in skin surface temperature $\left(500^{\circ} \mathrm{C} / \mathrm{sec}\right)$ (Haimi-Cohen et al. 1983) exciting intraepidermal nociceptive endings and eliciting a synchronous volley of afferent discharges that allows the recording of evoked potentials over the scalp. Subsequent human and animal studies have provided evidence that infrared laser-evoked potentials (LEPs) are due to the specific and synchronous activation of cutaneous $\mathrm{A} \delta$ and $\mathrm{C}$ afferent fibers and that LEP amplitudes are positively correlated with the intensity of the evoked pain sensation (Carmon et al. 1976, 1978; Devor et al. 1982; Kakigi et al. 1989). Similar results have been obtained with argon lasers (Arendt-Nielsen 1990).

Clinical application of the LEP has been limited by the size, expense, and relative immobility of lasers and by a lack of information about the sources of variability within individual subjects. For example, the effect of arousal and attention on the late components of the visual, auditory and somatosensory evoked potentials has been studied (Picton and Hillyard 1988), but com- 
parable data are not available for the LEP. Furthermore, LEP studies of individual patients with focal abnormalities of pain and temperature sensation (Casey et al. 1989; Bromm et al. 1991; Kakigi et al. 1991a: Treede et al. 1991) would be facilitated by information about the normal intrasubject variability of LEPs elicited by stimulating different body sites. We here report the results of LEP studies of normal human subjects in which a portable surgical infrared $\mathrm{CO}_{2}$ laser was used as a stimulus source. We reported previously on the effects of varying stimulus pulse duration and beam diameter on detection and pain thresholds using this laser (Pertovaara et al. 1988). In this study, we evaluated the effect of drowsiness, sleep and distraction on LEP amplitudes, analyzed perceived and applied stimulus intensity as correlates of LEP parameters, and analyzed normal intersubject and intrasubject lateralized (side-to-side) differences in LEP parameters as a basis for future clinical studies.

\section{Methods}

\section{Normative data}

Twenty healthy adults ( 18 men and 2 women) 20-40 years old with heights ranging from 165 to $185 \mathrm{~cm}$ served as subjects after signing an informed consent. Participation in this study was in accord with the International Association for the Study of Pain guidelines for human subjects. Both the subjects and experimenters wore protective goggles during data acquisition. Subjects were awake and relaxed with eyes closed in a reclined chair in a quiet room kept at $21-23^{\circ} \mathrm{C}$. The stimulus was a $60 \mathrm{msec}$ laser pulse (wave length $10.6 \mu \mathrm{m}$ ) generated by a $\mathrm{CO}_{2}$ surgical laser (Model 20, Directed Energy Inc.). After the subjects were acclimated to the experimental procedures, the dorsum of each hand was stimulated independently at intervals of 5-30 sec with a beam diameter adjusted to $6 \mathrm{~mm}(28$ $\mathrm{mm}^{2}$ ). The right and left hands of each subject were stimulated with identical stimulus intensities adjusted to a level that elicited a sharp pain similar to a pinprick (range: $0.14-0.21 \mathrm{~W} / \mathrm{mm}^{2}$. To avoid sensitization, beam location was moved slightly for each stimulus. The switch activating the laser system was muffled with a cloth to eliminate possible cueing of stimulus onset.

The LEPs were recorded with silver disks from $\mathrm{C} 4$, $\mathrm{C} 3$ and $\mathrm{Cz}$ (central vertex) referenced to linked ears (A1A2) with a ground at Pz. Amplifier gain was 30,000, and amplifier bandpass was $0.4-40 \mathrm{~Hz}(-3 \mathrm{~dB})$ with filter roll-off slopes of $12 \mathrm{~dB} /$ octave. Data were sampled at a rate of $512 \mathrm{~Hz}$ for $1 \mathrm{sec}$ following stimulus onset. Amplitude resolution of $\mathrm{A} / \mathrm{D}$ conversion was 8 bits. Electrode impedances were maintained between 1000 and $3000 \Omega$. The averaging process and raw EEG signals were displayed on an oscilloscope to monitor artifacts, with an on-line automatic rejection based on the amplitude of the responses. The responses to single trials were individually stored and subsequently reviewed for artifacts before averaging. The data were plotted on an X-Y plotter (Hewlett-Packard 7040B) and stored on floppy disks for subsequent analysis. Each average consisted of 25 samples, repeated at least once to ensure reproducibility of the potentials.

All amplitude and latency measures were calculated from the responses obtained at the $\mathrm{Cz}$ location because in all subjects the wave forms were best defined and highest in amplitude at this location. Absolute latencies were measured at the highest peak of each response component, while peak amplitudes were measured from baseline to peak. These measures were made from a grand average of reproducible wave forms from each side. Relative positivity at input terminal 2 resulted in a downward deflection in all records.

The means, standard deviations and coefficients of variability of peak latencies, peak amplitudes, interpeak latencies and interpeak amplitudes were obtained. Mean absolute and relative side-to-side differences were calculated for peak and interpeak latencies and amplitudes. The relative side-to-side differences were calculated according to the following formula:

[( higher value - lower value $) /$ higher value] $\times 100$.

\section{Conduction velocity determination}

At a single session, the feet and thighs of 6 normal subjects were unilaterally stimulated with laser pulses at a stimulus intensity adjusted to a level that elicited a sharp pain similar to a pinprick. The conduction velocity of the stimulated afferent fibers was determined by dividing the distance between the 2 stimulus sites by the peak latency difference of the early negative component.

\section{Correlations between perceived stimulus intensity and amplitudes or latencies of LEP components}

Five normal volunteers were the subjects of this experiment. They were stimulated at various laser intensities ranging between 10 and $50 \%$ of maximal laser power output and corresponding to $0.07-0.35 \mathrm{~W} / \mathrm{mm}^{2}$. The subjects received 25 stimuli at each stimulus intensity and were asked to rate each stimulus on a scale of $0-3(0=$ no sensation, $1=$ warm or hot sensation, $2=$ pinprick sensation, $3=$ pinprick sensation followed by a burning sensation). Because of the intersubject differences in LEP amplitudes and latencies, we computed the amplitude and latency for each subject and at each intensity as a percent of the maximal value recorded from that subject. The relationship between the amplitudes and latencies of the components with the perceived intensity and applied stimulus intensity was calculated with the Spearman rank correlation. 


\section{Effect of different states of arousal on LEPS}

Five normal subjects were sleep deprived 1 day before the study for this experiment. LEPs were obtained from each subject while lying down with eyes closed during wakefulness, drowsiness and stage 2 sleep. The stimulus intensity used throughout the experiment was adjusted to a level that elicited, in wakefulness, a sharp pain similar to a pinprick sensation. Eight channels of EEG and eye movements were monitored throughout the study with silver electrodes placed at the outer canthi, infraorbital, central and occipital positions referenced to ipsilateral ear. Onset of drowsiness was defined by a drop-out of alpha activity with slow lateral eye movements whereas stage 2 sleep was determined by the presence of sleep spindles in the central derivations (Rechtschaffen and Kales 1968). When a stimulus delivered during drowsiness or stage 2 sleep caused an arousal response, the subject was not stimulated until drowsiness or stage 2 sleep resumed. Each average consisted of 20 samples, repeated at least once to ensure reproducibility of the potentials. Comparison of LEP latencies and amplitudes during different states of arousal was performed with paired $t$ tests.

\section{Effect of distraction on the LEPS}

Five normal subjects participated in this experiment. During the first part of the experiment, LEPs were obtained while subjects concentrated on rating the perceived intensity of each of the laser pulses. During the second part of the experiment, the subjects were asked to solve 3 digit subtraction problems. While the subjects were solving the equation mentally, a laser pulse was delivered. The subjects were then asked to provide the answer to the problem. Accuracy of the responses was recorded and the test accepted if at least $60 \%$ of the responses were accurate. Each average consisted of 20 samples, repeated at least once to ensure reproducibility of the potentials. Comparison of LEP latencies and amplitudes during attention to and distraction from the stimuli was performed with paired $t$ tests.

\section{Results}

Scalp recordings demonstrated good definition and coherence of the potentials from all subjects on stimulation of either hand. A representative recording with major wave form components specified is shown in Fig. 1. This wave form is diphasic and consists of an earlier negativity with a peak latency close to $250 \mathrm{msec}$ (N250) followed by a positive component with a peak latency of about 350 msec (P350). In all subjects, an LEP was identifiable in most of the single averages.

\section{Effects of repetitive stimulation on the laser evoked potential}

There was no significant effect of repetitive stimulation on LEP amplitudes or latencies when up to 50 stimuli were averaged. The responses to the first 25 stimuli of 50 are nearly identical to the last 25 stimuli (Fig. 2).

\section{Conduction velocity determination}

The morphology of the LEPs following stimulation of the foot or thigh was similar to that obtained following hand stimulation (Fig. 3). The distance between the stimulated sites on the feet and thighs of 6 normal subjects averaged $82.8 \pm 2.4 \mathrm{~cm}$ (S.E.M.). The grand average peak latency of the negative component of the 6 subjects following foot stimulation was $278 \pm 13 \mathrm{msec}$ and following thigh stimulation was $220 \pm 9 \mathrm{msec}$. The peripheral conduction velocity of the afferent fibers activated by the laser pulses averaged $14.9 \pm 1.5 \mathrm{~m} / \mathrm{sec}$ (range $9.8-20.7 \mathrm{~m} / \mathrm{sec}$ ).

Correlations between perceived stimulus intensity and amplitudes or latencies of LEP components

Highly significant correlations were obtained between perceived intensities and percent maximal peak amplitudes of the $\mathrm{N}(r=0.71, P=0.0001)$ and $\mathrm{P}(r=$ $0.76, P<0.0001)$ components as well as with the percent maximal peak-to-peak values $(r=0.77, P<$ $0.0001)$. The correlation between percent maximal

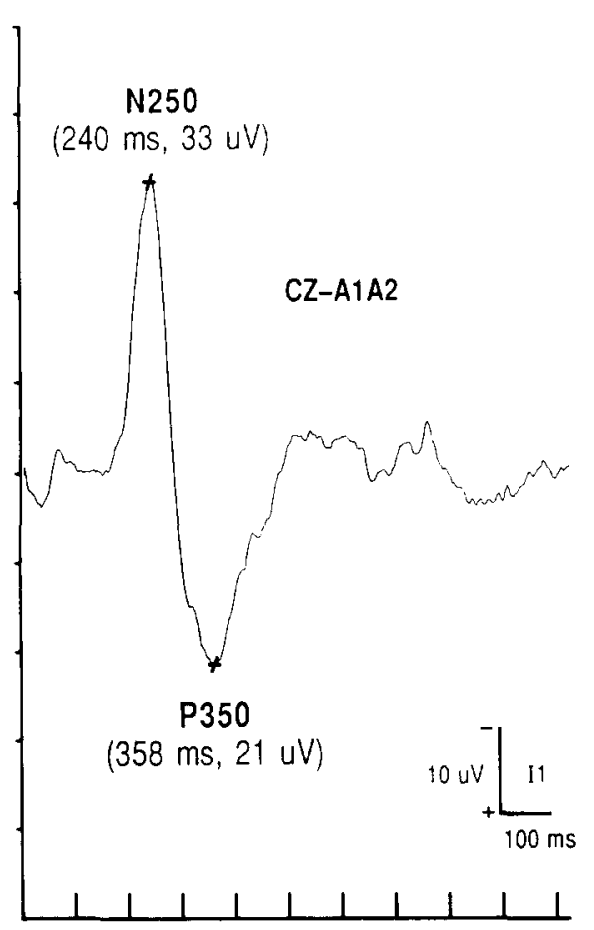

Fig. 1. Representative example of laser evoked potential recorded from a normal subject. Twenty-five stimuli were delivered to the dorsum of the hand at an intensity of $0.2 \mathrm{~W} / \mathrm{mm}^{2}$. 

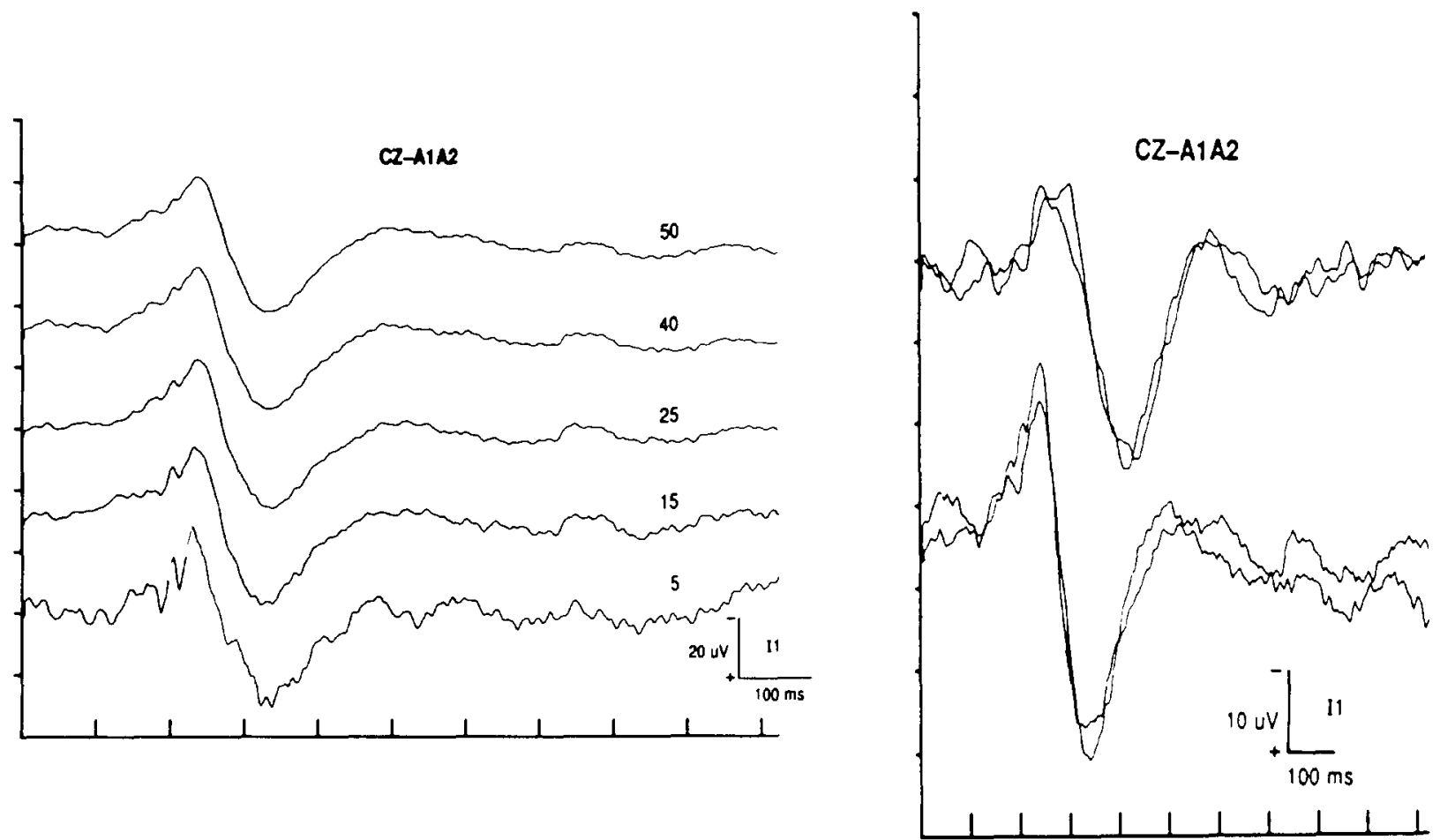

Fig. 2. Effect of repetitive stimulation on LEPs. Left: typical LEP from one subject as a function of the number of responses averaged. In all subjects, averaging 25 responses yielded a good signal-to-noise ratio. Right: the overlap of averaged responses to the first 25 and last 25 stimuli of 50 in 2 normal subjects are nearly identical, showing a lack of significant effect of repetitive stimulation when up to 50 stimuli are averaged.

peak-to-peak amplitude and perceived stimulus intensity for all subjects is shown in Fig. 4. The percent maximal amplitudes of the $\mathrm{N}$ and $\mathrm{P}$ components and

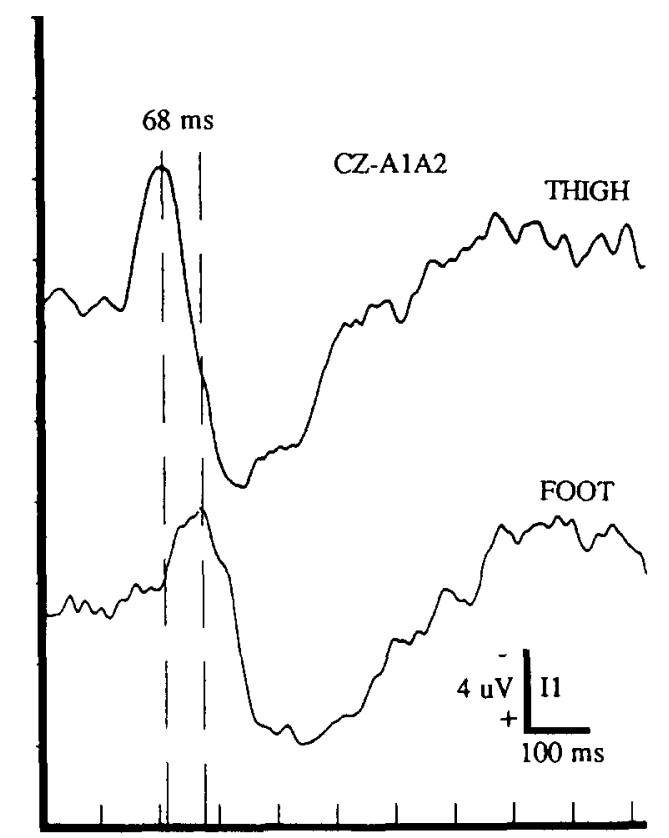

Fig. 3. Determination of the conduction velocity of afferent fibers in a normal subject. The difference in peak latency of the early negative component following foot and thigh stimulation was $68 \mathrm{msec}$. A distance of $86 \mathrm{~cm}$ separates the 2 sites of stimulation, yielding a conduction velocity of $12.6 \mathrm{~m} / \mathrm{sec}$. the peak-to-peak amplitudes correlated significantly although less strongly with applied stimulus intensity, with correlation coefficients of $0.67(P=0.002), 0.62$ $(P=0.004)$ and $0.68(P=0.001)$ respectively. In addition, there was a significant correlation between per-

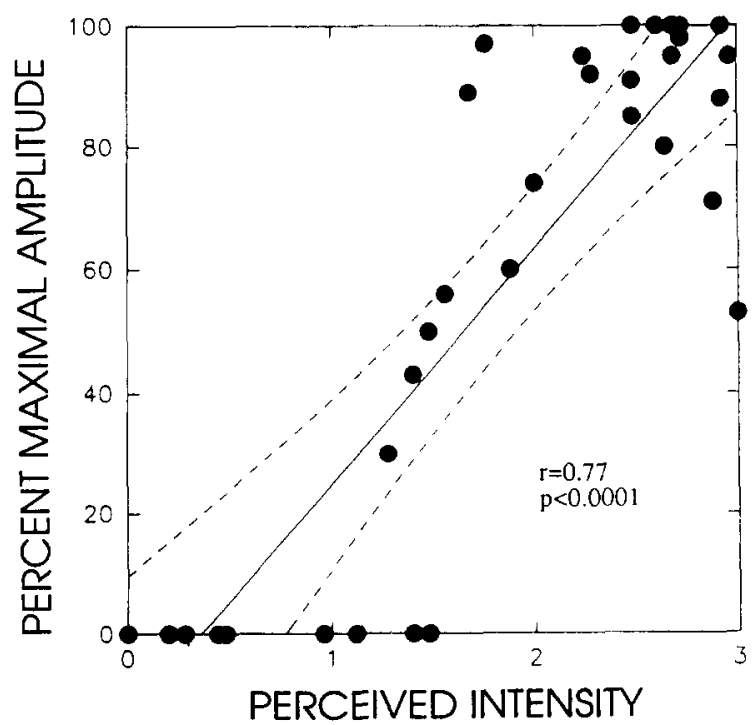

Fig. 4. Regression line of percent maximal peak-to-peak amplitude of LEPs versus perceived intensity in 5 normal subjects. The subjects were stimulated at various laser intensities ranging between 0.07 and $0.35 \mathrm{~W} / \mathrm{mm}^{2}$ and rated perceived intensity on a scale of $0-3(0=$ no sensation, $1=$ warm or hot sensation, $2=$ pinprick sensation, $3=$ pinprick sensation followed by a burning sensation). 

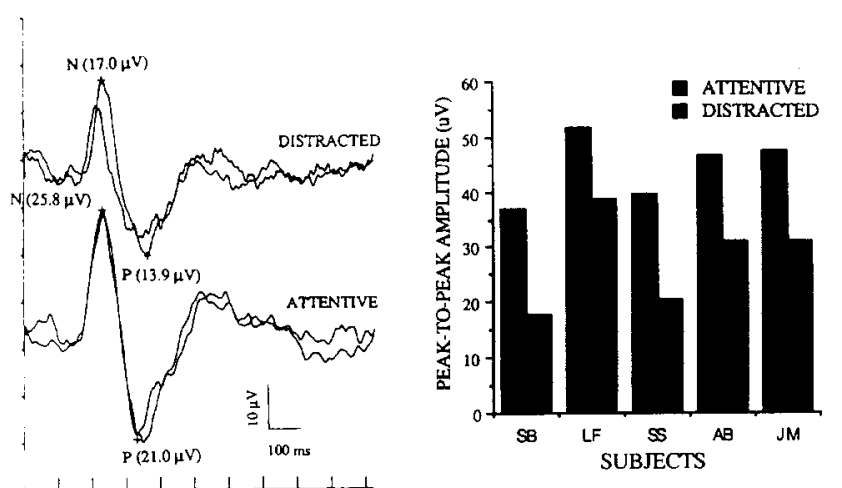

Fig. 5. Effect of distraction on the LEP. Left: the left hand of a normal subject was stimulated at $0.18 \mathrm{~W} / \mathrm{mm}^{2}$ while attentive to and when distracted from the stimulus. The reduction in LEP amplitude during distraction is evident. Latencies and amplitudes represent the average of the 2 overlapping trials. Right: the peak-to-peak amplitude of LEP during attentiveness and distraction in the 5 normal subjects.

ceived stimulus intensity and applied stimulus intensity ( $r=0.84, P<0.0001$ ).

There was a significant negative correlation between perceived intensity and percent maximal peak latency of the $\mathrm{N}$ component $(r=-0.62, P=0.003)$, but no significant correlation with the percent maximal latency of the $\mathrm{P}$ component or the interpeak latency $(P>0.05)$.

\section{Effect of distraction}

The average amplitudes of the $\mathrm{N}$ and $\mathrm{P}$ components during attentiveness to the stimulus were 19.9 and 24.6 $\mu \mathrm{V}$, respectively and the peak-to-peak amplitude was $44.5 \mu \mathrm{V}$. During distraction, the amplitude of these components decreased to a mean of 11.0, 16.8 and 27.7 $\mu \mathrm{V}$, respectively. These values correspond to a decrease of $45 \%, 39 \%$ and $38 \%$ of the amplitudes during attentiveness to the stimulus. These differences were statistically significant for all 3 amplitude measures (paired $t$ test; $P<0.05$ ). Fig. 5 illustrates this result and shows the effect of distraction in one subject. The peak latency of the $\mathrm{N}$ and $\mathrm{P}$ components averaged 236 and $366 \mathrm{msec}$ during attentiveness and 221 and 356 msec during distraction. None of these differences was statistically significant. Although the design of the ex-
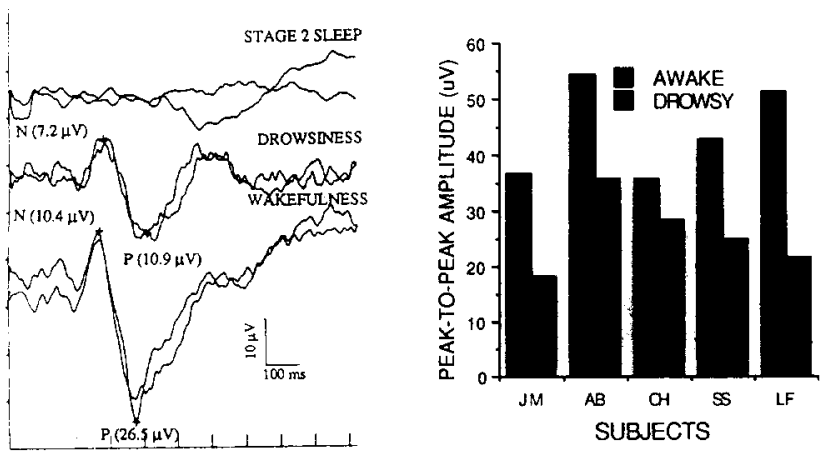

Fig. 6. Effect of levels of aoursal on the LEP. Left: the left hand of a normal subject was stimulated at $0.14 \mathrm{~W} / \mathrm{mm}^{2}$ during wakefulness, drowsiness and stage 2 sleep. The amplitude of the LEP is reduced during drowsiness and absent during stage 2 sleep. Latencies and amplitudes represent the average of the 2 overlapping trials. Right: the peak-to-peak amplitude of LEP during wakefulness and drowsiness in the 5 normal subjects.

periment precluded the subjects' rating the stimulus intensity, all subjects stated that the perceived intensity during distraction was of a lesser magnitude than when they concentrated on the stimulus.

\section{Effect of drowsiness and sleep}

The average amplitudes of the $\mathrm{N}$ and $\mathrm{P}$ components during wakefulness were 18.2 and $26.0 \mu \mathrm{V}$ respectively and the peak-to-peak amplitude was $44.3 \mu \mathrm{V}$. During drowsiness, the amplitude of these components decreased to $13.9,11.9$ and $25.9 \mu \mathrm{V}$ respectively. These values correspond to a decrease of $24 \%, 54 \%$ and $42 \%$ of the amplitudes during wakefulness. These were statistically significant differences for the $\mathrm{P}$ component amplitude and the peak-to-peak amplitude (paired $t$ test; $P<0.05$ ) but not for the amplitude of the $\mathrm{N}$ component $(P=0.08)$. Fig. 6 illustrates this result and shows the effect of drowsiness and sleep in one subject. The peak latencies of the $\mathbf{N}$ and $\mathbf{P}$ components averaged 229 and $355 \mathrm{msec}$ during wakefulness and 253 and 394 msec during drowsiness. Even though there was a trend toward prolongation of peak latencies during drowsiness, it did not reach statistical significance. In all subjects, the LEPs were absent during stage 2 sleep. Following some stimuli, the subjects

\section{TABLE I}

Peak and interpeak latencies and amplitudes of LEP components obtained from 20 normal subjects following stimulation of the dorsum of each hand. The last column shows values of mean + 3 S.D.s. S.D.: standard deviation; CV (\%): coefficient of variability.

\begin{tabular}{|c|c|c|c|c|c|}
\hline & Mean & S.D. & $\mathrm{CV}(\%)$ & Range & Mean + 3 S.D. \\
\hline Peak latency N (msec) & 233 & 21 & 9.0 & $185 \quad-267$ & 296 \\
\hline Peak latency P (msec) & 369 & 27 & 7.3 & $334-453$ & 450 \\
\hline Interpeak latency (msec) & 136 & 28 & 20.6 & $88-186$ & 220 \\
\hline Peak amplitude $N(\mu \mathrm{V})$ & 15.8 & 8.1 & 51.3 & $6.2-38.0$ & \\
\hline Peak amplitude $\mathrm{P}(\mu \mathrm{V})$ & 25.8 & 14.0 & 54.3 & $7.8-70.1$ & \\
\hline Interpeak amplitude $(\mu \mathrm{V})$ & 41.6 & 18.6 & 44.7 & $16.2-91.6$ & \\
\hline
\end{tabular}


TABLE II

Absolute (msec for latency and $\mu \mathrm{V}$ for amplitude) and relative (\%) side-to-side differences for peak and interpeak latencies and amplitudes of LEP components in 20 normal subjects following stimulation of the dorsum of each hand. The last column shows values of mean +3 S.D.s.

\begin{tabular}{|c|c|c|c|c|c|c|c|}
\hline & \multicolumn{3}{|c|}{ Absolute } & \multicolumn{3}{|c|}{ Relative } & \multirow[t]{2}{*}{ Mean + 3 S.D. } \\
\hline & Mean & S.D. & Range & Mean & S.D. & Range & \\
\hline Peak latency $\mathbf{N}$ & 7.5 & 9.5 & $0-42$ & 3.3 & 4.5 & $0-20.4$ & \\
\hline Peak latency $P$ & 11.9 & 8.1 & $\begin{array}{ll}4 & -38\end{array}$ & 3.0 & 2.2 & $0-9.9$ & \\
\hline Interpeak latency & 10.7 & 8.2 & $0-26$ & 7.7 & 5.5 & $0-16.9$ & \\
\hline Peak amplitude $\mathrm{N}$ & 2.2 & 2.4 & $0.3-9.9$ & 13.8 & 12.7 & $1.5-42.3$ & 51.9 \\
\hline Peak ampltiude $P$ & 3.0 & 2.6 & $0.1-9.3$ & 11.4 & 9.1 & $0.5-28.1$ & 38.7 \\
\hline Interpeak amplitude & 3.7 & 2.9 & $0-10.6$ & 8.9 & 6.5 & $0-22.1$ & 28.4 \\
\hline
\end{tabular}

developed a vertex wave or a $\mathrm{K}$ complex following stimulation. From review of the single trial averages, none of the subjects had a detectable LEP following any of the stimuli delivered.

\section{Intrasubject lateralized differences}

The mean peak and interpeak latencies and amplitudes of the N250 and P350 components are shown in Table I. There was no significant difference in the results obtained from right or left hand stimulation, so they were combined. There was considerable intersubject variability of peak latencies and amplitudes of the $\mathrm{N}$ and $\mathrm{P}$ components as evidenced by the wide ranges and high coefficients of variability (Table I). To obtain a measure of intrasubject variability, the absolute and relative side-to-side differences for peak and interpeak latencies and amplitudes were calculated (Table II). The relative interpeak amplitude difference was less variable than the relative amplitude of the $N$ or $P$ components. In setting a 3 S.D. criterion for the detection of focal or lateralized abnormality in clinical studies, a side-to-side interpeak amplitude difference greater than $28 \%$ would be considered abnormal (Table II).

\section{Discussion}

Since the original description by Carmon et al. (1976), various investigators have studied the evoked potentials generated by cutaneous stimulation with radiant heat pulses (Kenton et al. 1980; Treede et al. 1988; Kakigi et al. 1989). The stimulation and recording techniques have varied between laboratories, making a comparison of results difficult. We recorded a reproducible biphasic (negative-positive) potential complex from all subjects. The N250 and P350 components correspond to the $\mathrm{N} 2$ and $\mathrm{P} 2$ components reported by Treede et al. (1988) and to the N200 and P320 reported by Kakigi et al. (1989). The peak latencies of the biphasic potential described by Carmon et al. (1978) were shorter than ours because of their more proximal stimulation site, the lateral aspect of the forearm.

We found that the peripheral afferent fibers activated by the laser pulses have an average conduction velocity of $14.9 \mathrm{~m} / \mathrm{sec}$, which is within the range of human A $\delta$ fibers (Vallbo et al. 1979; Adriaensen et al. 1983). Similar results were reported by Bromm and Treede (1987), who stimulated the arm and hand in 11 normal subjects and found an average conduction velocity of $16.1 \mathrm{~m} / \mathrm{sec}$, by Kenton et al. (1980), who found an average conduction velocity of $10.8 \mathrm{~m} / \mathrm{sec}$, and recently by Kakigi et al. (1991b), who reported a conduction velocity of approximately $9 \mathrm{~m} / \mathrm{sec}$ for both upper and lower extremities. Microneurographic studies in humans (Bromm et al. 1984) and animals (Devor et al. 1982) have found that $\mathrm{CO}_{2}$ laser stimuli activated $\mathrm{A} \delta$ and polymodal $\mathrm{C}$ fibers. If conduction of $\mathrm{A}$ fibers was blocked by pressure, the $\mathrm{A} \delta$-related cerebral potentials disappeared (Bromm and Treede 1984). Because $A \delta$ fibers innervate nociceptors (Bishop and Landau 1958; Sinclair and Stokes 1964), these are important findings in establishing a relationship between the LEPs and nociceptive input.

The peak and interpeak amplitudes of the $\mathrm{N}$ and $\mathrm{P}$ components correlated significantly with both perceived intensity and applied stimulus intensity. Because increasing the stimulus intensity usually leads to progressively higher magnitude of perception, it is difficult to determine if the LEP amplitudes better reflect applied stimulus intensity or perceived intensity. Similar findings using discrete pain rating scales were reported by Carmon et al. (1978) using laser pulses and by Chen et al. (1979) using dental electrical stimulation. Our findings differ from the results of Carmon et al. (1978), who found a significant correlation with the amplitude of the positive but not the negative component of the LEPs. More studies are needed to determine if this relationship is linear throughout the entire range of perception or only over a specific range of pain perception, as suggested by Arendt-Nielsen (1990). Perhaps differences in technique and in the conditions of data acquisition could uncouple the relationship between 
perceived intensity and LEP amplitudes. For example, Chapman et al. (1981) found that the amplitude of a potential evoked by electrical stimulation of the tooth pulp varied with stimulus frequency while the subject's pain ratings were unchanged. Although laser pulse stimulation of the skin cannot easily be compared with electrical stimulation of the tooth pulp, it is possible that neurophysiological factors other than those related to perceived intensity can affect the amplitude of LEPs or that the correlation applies only for a specific range of perceived pain sensations.

To uncouple the relationship between perceived intensity and applied stimulus intensity during wakefulness, we stimulated subjects at the same intensity during attentiveness to and distraction from the stimulus. We found that the amplitude of the $\mathrm{N}$ and $\mathrm{P}$ components as well as the peak-to-peak amplitude were significantly reduced when the subjects were distracted from the stimulus. It is important to note that the $P$ component of LEPs was found to be totally unrelated to the P300 oddball potential (Towell and Boyd 1992). Although the design of this experiment precluded the subjects' rating each stimulus, all stated that the perceived intensities they experienced when distracted were of a lesser magnitude than when concentrating on the stimulus. This result shows that stimulus intensity is not the sole or perhaps even the major determinant of LEP amplitudes and suggests that these amplitudes may more closely reflect the perceived intensity in normal subjects. Similar reduction in the amplitude of long latency potentials has been reported in the visual (Hillyard et al. 1984), auditory (Okita 1979) and somatosensory (Desmedt and Robertson 1977; Miltner et al. 1989) modalities when distracted from incoming stimuli. Although the neural mechanisms underlying attention-related modulation of sensory processes is not well understood, it was suggested by Picton and Hillyard (1988) that stimuli not attended to may be filtered out or attenuated at an early stage of sensory processing before the perceptual analysis is complete. This could occur through a modulation through the thalamus (Hillyard et al. 1984) or reduction of information processing in the cortex (Wurtz et al. 1980). The significant effect of distraction on LEP amplitudes is consistent with previous studies documenting a reduction in pain perception when subjects are distracted (Clum et al. 1982; Spanos et al. 1984) and underscores the importance of having the subjects be attentive to the stimulus and to rate each stimulus when acquiring these potentials.

The state of arousal had a significant effect on the amplitude of LEPs. Indeed, the responses were undetectable during stage 2 sleep and were significantly reduced during drowsiness compared to the awake state. An explanation for the absence of the P350 component during stage 2 sleep could be the occa- sional occurrence of a negative potential with a peak latency of $300-500 \mathrm{msec}$ following stimulation and representing a vertex wave (Ornitz et al. 1967). To assess this possibility, we reviewed all single trials performed during stage 2 sleep and on no occasion was an LEP identified, even in the many instances when a vertex potential was absent. This result demonstrates that the responsiveness to laser stimulation is modulated during drowsiness and especially during sleep.

Because fluctuations in level of arousal and attentiveness to the stimulus can have a significant effect on LEPs, it is important to perform this test as quickly as possible and to monitor these variables. We found that, in cooperative subjects, averaging 25 responses gave a good signal-to-noise ratio, a result similar to that reported by Arendt-Nielsen (1990) using an argon laser. Our data also indicate that the effect of repetitive stimulation was minimal when up to 50 stimuli were averaged. Kakigi et al. (1989) averaged from 50 to 400 responses and used a short, invariable interstimulus duration of $3 \mathrm{sec}$, stimulus features that might introduce habituation (Angel et al. 1985) and expectation (Schafer et al. 1981) which may contribute to the fact that the $\mathrm{N}$ and $\mathrm{P}$ amplitudes they reported were 5-15 times smaller than those we recorded.

To evaluate the clinical utility of LEPs in patients with abnormalities of pain and/or temperature sensation, it is necessary to establish a set of normative data. In a study of LEPs in 18 patients with dissociated sensory loss, Bromm et al. (1991) chose a 50\% side-toside difference in peak-to-peak amplitude or peak latency as the upper limit of normal. Using these criteria, they found the LEPs to be abnormal on the pathological side in 14 out of the 18 patients studied. If a lateralized difference in amplitude of $28 \%$ or greater was considered abnormal, 3 additional patients would have been considered to have abnormal LEPs on the pathological side. When testing patients, some of whom will have decreased or absent cutaneous pain sensation, it is important to set an upper limit for stimulus intensity in order to prevent cutaneous damage. In this study, we found that an intensity range of 0.14-0.21 $\mathrm{W} / \mathrm{mm}^{2}$ elicited painful sensations in all normal subjects and produced only delayed and temporary discoloration in the stratum corneum (Treede et al. 1991). We also found that an intensity of up to $0.35 \mathrm{~W} / \mathrm{mm}^{2}$ did not result in any further skin damage and would use this intensity as the upper limit when testing patients. Our results show that LEPs exhibit great intersubject variability for peak and peak-to-peak amplitudes and latencies. Remarkably similar results for mean peak and interpeak latencies and respective standard deviations were reported by Treede et al. (1988). These investigators also found mean peak amplitudes of $5 \mu \mathrm{V}$ for the negative and $17 \mu \mathrm{V}$ for the positive components; they did not report side-to-side differ- 
ences. We found substantial intraindividual variability for side-to-side differences of peak and interpeak latencies and amplitudes (Table II). However, when the data were normalized to percentage differences, we found that the peak-to-peak amplitude was the least variable of the amplitude measures we compared and thus could be useful in the investigation of lateralized or focal pathology of the pain and temperature pathways. Because of the high interindividual variability of the amplitude and latency of these responses, the clinical study of individual patients is best applied to lateralized comparisons.

The authors thank Dr. Ivo Drury for review of the manuscript and Miss Sally Mouilleseaux for assistance in its preparation.

Supported by the Department of Veterans Affairs and an unrestricted award from Brystol-Myers to KLC.

\section{References}

Adriaensen, H., Gybeis, J., Handwerker, H.O. and Van Hees, J. Response properties of thin myelinated (A-delta) fibers in human skin nerves. J. Neurophysiol., 1983, 49: 111-122.

Angel, R.W., Quick, W.M., Boylls, C.C., Weinrich, M. and Rodnitzky, R.L. Decrement of somatosensory evoked potentials during repetitive stimulation. Electroenceph. clin. Neurophysiol., 1985, 60: 335-342.

Arendt-Nielsen, L. First pain event related potentials to argon laser stimuli: recording and quantification. J. Neurol. Neurosurg. Psychiat., 1990, 53: 398-404.

Bishop, G.H. and Landau, W.M. Evidence for a double peripheral pathway for pain. Science, 1958, 128: 712-713.

Bromm, B. and Treede, R.D. Nerve fibre discharges, cerebral potentials and sensations induced by $\mathrm{CO}_{2}$ laser stimulation. Hum. Neurobiol., 1984, 3: 33-40.

Bromm, B. and Treede, R.D. Pain related cerebral potentials: late and ultralate components. Int. J. Neurosci., 1987, 33: 15-23.

Bromm, B., Jahnke, M.T. and Treede, R.D. Responses of human cutaneous afferents to $\mathrm{CO}_{2}$ laser stimuli causing pain. Exp. Brain Res., 1984, 55: 158-166.

Bromm, B., Frieling, A. and Lankers, J. Laser-evoked potentials in patients with dissociated loss of pain and temperature sensibility. Electroenceph. clin. Neurophysiol., 1991, 80: 284-291.

Carmon, A., Mor, J. and Goldberg, J. Evoked cerebral responses to noxious thermal stimuli in humans. Exp. Brain Res., 1976, 25: 103-107.

Carmon, A., Dotan, Y. and Sarne, Y. Correlation of subjective pain experience with cerebral evoked responses to noxious thermal stimulations. Exp. Brain Res., 1978, 33: 445-453.

Casey, K.L., Huang, G.C.H. and Morrow, T.J. The role of cortical, thalamic and subthalamic neurons in pain: evidence from laser evoked potentials and sensory testing in patients and normal subjects. Soc. Neurosci. Abst., 1989, 15: 1190.

Chapman, C.R., Chen, A.C.N. and Harkins, S.W. Brain evoked potentials as correlates of laboratory pain: a review and perspective. In: J.J. Bonica, J.C. Liebeskind and D.G. Albe-Fessard (Eds.), Advances in Pain Research and Therapy, Vol. 3. Raven Press, New York, 1979: 791-803.

Chapman, C.R., Colpitts, Y.M., Mayeno, J.K. and Gagliardi, G.J. Rate of stimulus repetition changes evoked potential amplitude: dental and auditory modalities compared. Exp. Brain Res., 1981, 43: $246-252$.
Chatrian, G.E., Canfield, R.C., Knauss, T.A. and Lettich, E. Cerebral responses to electrical tooth pulp stimulation in man: an objective correlate of acute experimental pain. Neurology, 1975, 25: 745-757.

Chen, A.C.N., Chapman, C.R. and Harkins, S.W. Brain evoked potentials are functional correlates of induced pain in man. Pain, 1979, 6: 365-374.

Clum, G.A., Luscomb, R.L. and Scott, L. Relaxation training and cognitive redirection strategies in the treatment of acute pain. Pain, 1982, 12: 175-183.

Desmedt, J.E. and Robertson, D. Differential enhancement of early and late components of the cerebral somatosensory evoked potentials during forced-paced cognitive tasks in man. J. Physiol. (Lond.), 1977, 271: 761-782.

Devor, M., Carmon, A. and Frostig, R. Primary afferent and spinal sensory neurons that respond to brief pulses of intense infrared radiation: a preliminary servey in rats. Exp. Neurol., 1982, 76: 483-494.

Gybels, J., Handwerker, H.O. and Van Hees, J. A comparison between the discharges of human nociceptive nerve fibres and the subject's ratings of his sensations. J. Physiol. (Lond.), 1979, 292: 193-206.

Haimi-Cohen, R., Cohen, A. and Carmon, A. A model for the temperature distribution in skin noxiously stimulated by a brief pulse of $\mathrm{CO}_{2}$ laser radiation. J. Neurosci. Meth., 1983, 8: 127-137.

Hillyard, S.A., Simpson, G.V., Woods, D.L., Van Voorhis, S. and Munte, T. Event-related brain potentials and selective attention to different modalities. In: F. Reinoso-Suarez and C. Ajmone Marsan (Eds.), Cortical Integration. Raven Press, New York, 1984: 395-414.

Kakigi, R., Shibasaki, H. and Ikeda, A. Pain-related somatosensory evoked potentials following $\mathrm{CO}_{2}$ laser stimulation in man. Electroenceph. clin. Neurophysiol., 1989, 74: 139-146.

Kakigi, R., Shibasaki, H., Kuroda, Y., Neshige, R., Endo, C., Tabuchi, K. and Kishikawa, T. Pain-related somatosensory evoked potentials in syringomyelia. Brain, 1991a, 114: 1871-1889.

Kakigi, R., Endo, C., Neshige, R., Kuroda, Y. and Shibasaki, H. Estimation of conduction velocity of $\mathrm{A} \delta$ fibers in humans. Muscle Nerve, 1991b, 14: 1193-1196.

Kenton, B., Coger, R., Crue, B., Pinsky, J., Friedman, Y. and Carmon, A. Peripheral fiber correlates to noxious thermal stimulation in humans. Neurosci. Lett., 1980, 17: 301-306.

Miltner, W., Johnson, R., Braun, C. and Larbig, W. Somatosensory event-related potentials to painful and non-painful stimuli: effects of attention. Pain, 1989, 38: 303-312.

Okita, T. Event-related potentials and selective attention to auditory stimuli varying in pitch and localization. Biol. Psychol., 1979, 9: 271-284.

Ornitz, E.M., Ritvo, E.R., Carr, E.M., La Franchi, S. and Walter, R.D. THe effect of sleep onset on the auditory averaged evoked response. Electroenceph. clin. Neurophysiol., 1967, 23: 335-341.

Pertovaara, A., Morrow, T.J. and Casey, K.L. Cutaneous pain and detection thresholds to short $\mathrm{CO}_{2}$ laser pulses in humans: evidence on afferent mechanisms and the influence of varying stimulus conditions. Pain, 1988, 34: 261-269.

Picton, T.W. and Hillyard, S.A. Endogenous event-related potentials. In: T.W. Picton (Ed.), Human Event-Related Potentials. Handbook of Electroencephalography and Clinical Neurophysiology (revised series), Vol. 3. Elsevier, Amsterdam, 1988: 361-426.

Schafer, E.W.P., Amochaev, A. and Russell, M.J. Knowledge of stimulus timing attenuates human evoked cortical potentials. Electroenceph. clin. Neurophysiol., 1981, 52: 9-17.

Sinclair, D.C. and Stokes, B.A.R. The production and characteristics of 'second pain'. Brain, 1964, 87: 609-618.

Spanos, N.P., McNeil, C., Gwynn, M. and Stam, H.J. Effects of suggestion and distraction on reported pain. J. Abnorm. Psychol., 1984, 93: 277-284. 
Towell, A.D. and Boyd, S.G. The $\mathrm{CO}_{2}$-laser-evoked cerebral potential in humans is not a P300 oddball potential. J. Physiol. (Lond.), 1992, 452: 281P.

Treede, R.D., Kief, S., Holzer, T. and Bromm, B. Late somatosensory evoked cerebral potentials in response to cutaneous heat stimuli. Electroenceph. clin. Neurophysiol., 1988, 70: 429-441.

Treede, R.D., Lankers, J., Frieling, A., Zangemeister, W.H., Kunze, K. and Bromm, B. Cerebral potentials evoked by painful laser stimuli in patients with syringomyelia. Brain, 1991, 114: 15951607.
Vallbo, Å.B., Hagbarth, K.E., Torebjörk, H.E. and Wallin, B.G. Somatosensory, proprioceptive and sympathetic activity in human peripheral nerves. Physiol. Rev., 1979, 59: 919-957.

Wurtz, R.H., Goldberg, M.E. and Robinson, D.L. Behavioral modulation of visual responses in the monkey: stimulus selection for attention and movement. Progr. Psychobiol. Physiol. Psychol., 1980, 9: 43-83. 